\title{
Concepciones de los estudiantes de Magisterio sobre Moodle
}

\section{The future teachers' conceptions about Moodle}

\author{
Juan Carlos Rivadulla-López \\ Departamento de Pedagoxía e Didáctica. Universidade da Coruña
}

\begin{abstract}
Resumen
En este trabajo se pretende conocer la formación que tienen los estudiantes universitarios sobre la plataforma Moodle ay se analizarán los usos que hacen de ella y determinar las ventajas y las limitaciones que le otorgan. En la investigación han participado 81 estudiantes del Grado de Educación Infantil de la Universidade da Coruña y como instrumento se utilizó un cuestionario abierto. Los resultados muestran que los alumnos tienen carencias respecto al uso de Moodle, para lo cual demandan una may or formación específica.
\end{abstract}

Palabras clave: Moodle, método de enseñanza, enseñanza a distancia, alumnos

\begin{abstract}
This work intends to learn about training with college students on the platform Moodle ay analysed is applications that make it and determine the advantages and limitations that give. The research involved 81 students of the degree of child education from the Universidade da Coruña and an open questionnaire was used as instrument. The results show that students have shortcomings with respect to the use of Moodle, for which demand a more specific training.
\end{abstract}

Keywords: Moodle, teaching method, distance study, students

\section{Introducción}

El uso de las tecnologías de la información y la comunicación (TIC) y las tecnologías digitales y las plataformas de publicación web, han venido favoreciendo en los últimos años la modernización de los modelos educativos tradicionales basados, por un lado, en la presencialidad del alu mno y, por el otro, en la clase magistral del docente.

A ello hay que añadirle la integración de los centros universitarios españoles en el denominado Espacio Europeo de Educación Superior (EEES), lo cual supuso una profunda reflexión sobre las metodologías docentes a aplicar a fin de alcanzar el paradigma promulgado por este nuevo modelo de educación universitaria, en el que se pretende establecer un sistema basado en la adquisición de competencias y con una clara orientación hacia el aprendizaje del estudiante, sin excluir, con ello, el enfoque clásico basado en contenidos y horas lectivas (Martín y Rodríguez, 2012). El alumno ha de adquirir una serie de competencias y habilidades a través de un proceso de autoaprendizaje guiado y tutorizado, siendo, siendo su participación mucho más activa que la mera asistencia a clase o el desarrollo de alguna actividad de carácter práctico (Oliveros, 2006), aunque estos nuevos modelos de enseñanza no están exentos de problemas y de detractores (Ahn y Han, 2005).

A través de este nuevo concepto de aprendizaje de las enseñanzas universitarias se promueve la flexibilidad, como respuesta a las demandas de las sociedades actuales en un contexto abierto y en constante transformación, surgiendo nuevas modalidades formativas que recogen lo mejor del sistema tradicional y lo complementen con las nuevas técnicas y procedimientos propios de los modelos educativos no presenciales en entornos de red. Entre estas nuevas modalidades podemos destacar el b-learning (blended learning o aprendizaje mixto), el e-learning (electronic learning o aprendizaje electrónico o a distancia) o, más recientemente, el m-learning (mobile learning o aprendizaje electrónico móvil). En todos ellos, el elemento central para la construcción y la administración de las webs docentes y de las actividades de formación no presencial lo constituye una plataforma virtual de teleformación, a menudo referidas de innumerables maneras (Díaz-Antón y Pérez, 2005), pero habitualmente conocidas en su acepción anglosajona como CMS (Course Management System) o, de forma más habitual, LMS (Learning Management System).

Entre los retos que plantea este nuevo escenario destacamos el de avanzar hacia la incorporación y combinación progresiva de metodologías activas (MEC, 2006), donde las innovaciones tecnológicas favorecedoras de entornos virtuales de aprendizaje nos ofrecen mayores posibilidades pedagógicas (Bautista, Borges y Forés, 2006).

Actualmente, la mayoría de las universidades utilizan la información contenida en los ficheros de actividad de las plataformas de gestión para el aprendizaje a distancia para intentar medir el grado de calidad de las enseñanzas impartidas (Martín y Rodríguez, 2012). Entre estas plataformas, la más utilizada se puede decir que es Moodle, la cual es una de las plataformas más completas y adecuadas para su implementación en la Educación Superior (Aydin y Tirkes, 2010).

Moodle ofrece las herramientas necesarias para la docencia universitaria, tanto presencial, semipresencial o a distancia (Saito y Ulbricht, 2012; Williams Van Rooij, 2012) y promociona nuevos aprendizajes, facilitando el acceso al material de forma organizada (Peat y Franklin, 2002). Además, facilita el desarrollo de procesos de 
enseñanza-aprendizaje en la formación elearning, b-learning y presencial por razones como la interacción (Swan, Shea, Fredericksen, Pickett, Pelz y Maher, 2002), la usabilidad y la presencia social (Richardson y Swan, 2003).

La distribución de Moodle es gratuita debido a que se trata de software libre (Open Source) (Martín y Rodríguez, 2012) sujeto a la Licencia Pública GNU, lo cual le permite tener derechos de autor (copyright), dándole al usuario del software libertades como copiar, usar y modificar Moodle siempre que acepte proporcionar el código fuente a otros, no modificar o eliminar la licencia original y los derechos de autor, y aplicar esta mis ma licencia a cualquier trabajo derivado de él (Martínez y Fernández, 2011).

Adell, Castellet y Gu mbau (2004), después de analizar varios entornos virtuales de enseñanza-aprendizaje, recomiendan la utilización de Moodle por:

- Ofrecer unas funcionalidades didácticas sofisticadas y ricas en opciones. Su flexibilidad, derivada de su estructura modular, es lo que garantiza dar soporte a cualquier estilo docente.

- Disponer de más opciones (su carácter modular no dificulta su usabilidad).

- Brindar un grado de apertura y dinamismo del proyecto más elevados debido a la participación de desarrolladores de todo el mundo, lo que permite el mejoramiento de la plataforma a través de módulos y características adicionales disponibles de forma libre.

- Permitir la creación de espacios destinados a la enseñanza que en el contexto anglosajón se conocen como Virtual Learning Enviroments (VLE) o más recientemente Personal Learning Enviroments (PLE). En el contexto iberoamericano, se conocen como entornos virtuales de aprendizaje (EVA) o entornos virtuales de enseñanza aprendizaje (EVEA) y entornos de aprendizaje personalizados (EAP).

Además, numerosos estudios han demostrado que, aunque el alumnado no suele tener competencias en Moodle e incluso algunos no saben lo que es (Henríquez y Ugel, 2012), la implantación de esta plataforma educativa mejora significativamente el rendimiento mostrado por los alumnos (Martín y Serrano, 2009; Escobar y Monge, 2012), pues estos desarrollan el sentido de conectividad y de comunidad y aumenta su capacidad de aprendizaje (Perkins y Pfaffan, 2006).

Tal y como se pudo comprobar a lo largo de este apartado, son abundantes los estudios sobre la incorporacion de las TIC a la ensenanza universitaria, tanto a nivel nacional como internacional, y en concreto acerca del uso de Moodle. Sin embargo, son menos numerosos los estudios encaminados a analizarla percepcion del alumnado. Por todo ello, este trabajo muestra las percepciones que los estudiantes del Grado de Educación Infantil de la Universidade da coruña tienen sobre Moodle.

\section{Método}

Teniendo en cuenta que el interés principal en este estudio fue conocer y analizar las percepciones de un grupo de estudiantes de la Facultad de Ciencias de la
Educación de la Universidade da Coruña sobre los usos de la plataforma Moodle, así como determinar las ventajas y limitaciones que le otorgan a dicha plataforma, las condiciones que se propusieron para participar fueron que los estudiantes empezaran recientemente a usar la plataforma Moodle en la universidad y que hubiesen tenido más de cinco asignaturas con apoyo en la plataforma estudiada. Por estos dos motivos se escogió como población objeto de estudio a los 120 estudiantes matriculados en segundo curso del Grado de Educación Infantil, aunque la muestra final fue de 81 part icipantes (67.5\%), de los cuales la mayoría son mu jeres (92.6\%) de entre 19 y 25 años (71.6\%).

Tabla 1.

Características socio-demográficas de la muestra.

\begin{tabular}{ccc}
\hline \multicolumn{2}{c}{ Variables } & $\begin{array}{c}\text { Total } \\
(\mathbf{n}=\mathbf{8 1})\end{array}$ \\
\hline \multirow{2}{*}{ Género } & n a...nn & $75(92.6 \%)$ \\
& Hombre & $6(7.4 \%)$ \\
\hline \multirow{3}{*}{ Edad } & $19-55$ & $58(71.6 \%)$ \\
& $26-30$ & $16(19.8 \%)$ \\
& +31 & $7(8.6 \%)$ \\
\hline
\end{tabular}

\section{Instrumentos o Materiales}

El método utilizado fue el de encuesta con vocación descriptiva (Bisquerra, 2004). Para la recogida de información se ha utilizado un cuestionario validado mediante 2 jueces y una experiencia piloto, y está compuesto por 4 dimensiones de análisis: datos de identificación, formación en Moodle, usos de Moodle y valoración sobre Moodle. La respuesta a cada una de esas dimensiones era abierta, pudiendo aportar más de una respuesta en cada una de ellas.

Además, cabe indicar que la dimensión sobre "Uso de Moodle" es una adaptación reducida de la propuesta de Sánchez, Sánchez y Ramos (2012), del Cuestionario de usos pedagógicos de Moodle en la docencia universitaria desde la perspectiva de los estudiantes de la Universidad de Castilla-La Mancha; y también de la propuesta de Marín y Amentia (2009) para conocer la percepción de los estudiantes de la Universidad del País Vasco sobre el uso de Moodle y eKasi.

\section{Procedimiento}

La recogida de datos se desarrolló durante el segundo cuatrimestre del curso académico 2013-2014 y las respuestas se analizaron de forma directa, agrupándose las mismas en categorías en función de su similitud.

\section{Resultados}

El 37.1\% de los participantes en esta investigación afirman que han recibido algún tipo de formación específica relacionada con la plataforma Moodle, y todos 
ellos la recibieron exclusivamente en la Universidad. En relación a la formación específica que les gustaría recibir sobre Moodle (figura 1), a la mayoría le gustaría aprender a usar el correo electrónico (QuickMail) (44.4\%) y la barra de herramientas (38.3\%). También les gustaría recibir formación sobre el funcionamiento en general del Moodle (30.9\%), aprender sobre el uso de los foros y blogs para poder mantener un contacto más directo con el profes orado y poder resolver dudas $(22.2 \%$ en ambos casos) y a un porcentaje más reducido (16.1\%) le gustaría aprender a bajar archivos (16.1\%).

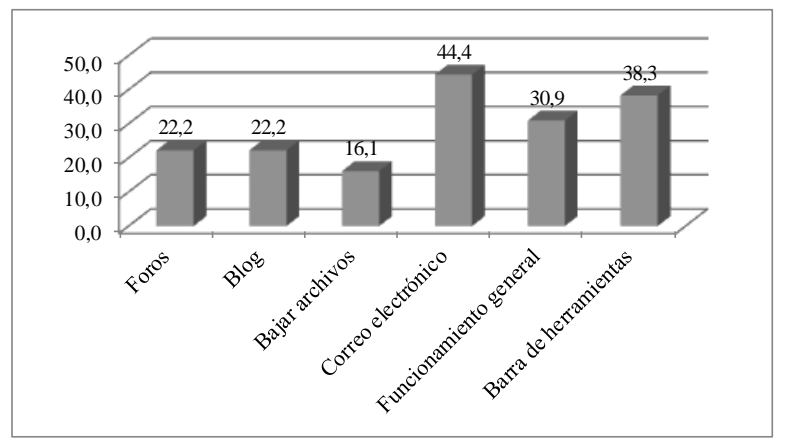

Figura 1. Formación que desearían recibir sobre Moodle

En cuanto a los usos que hacen de Moodle (figura 2), el total de los estudiantes del Grado de Educación Infantil consultados hacen referencia a la descarga de apuntes $(100 \%)$. Por su parte, ta mbién aluden alenvío de tareas al profesorado (37.1\%), a la lectura y envío de correos entre el profesor y el alumno (34.6\%) y a la lectura de los documentos que se cuelgan en la plataforma (33.3\%).

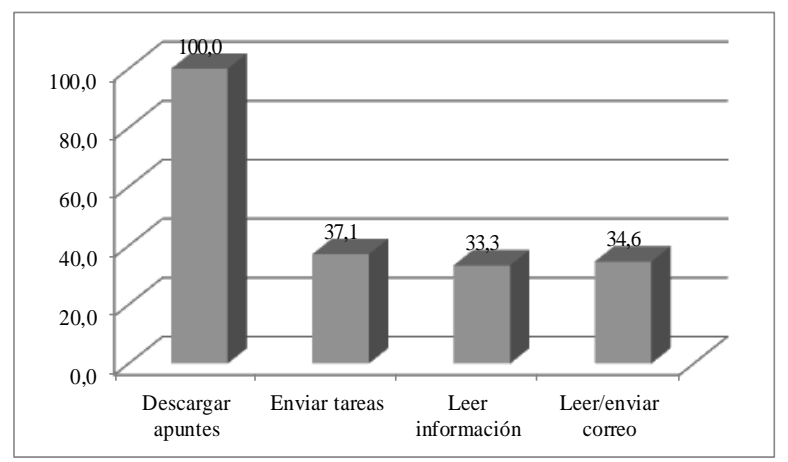

Figura 2. Usos de Moodle

Por último, a los alumnos consultados se les preguntó por las ventajas y las limitaciones que encontraban en el uso diario de la plataforma Moodle. Así, entre las principales ventajas destacadas por los estudiantes (figura 3), se pueden mencionar el ahorro económico que supone la no impresión de los documentos (55.5\%), la obtención y consulta ilimitada de los apuntes (45.7\% y $46.9 \%$ respectivamente). En menor medida, los alu mnos también se refieren a la facilidad para obtener información detallada de la asignatura (38.3\%), al ahorro de tiempo (35.8\%). Por otra parte, un porcentaje inferior de estudiantes hacen referencia a la posibilidad de mantener comunicación de manera rápida con el profes or
(24.7\%), a la facilidad para realizar entregas de trabajos a través de la plataforma $(22.2 \%)$ y para acceder desde cualquier lugar a Moodle (12.3\%).

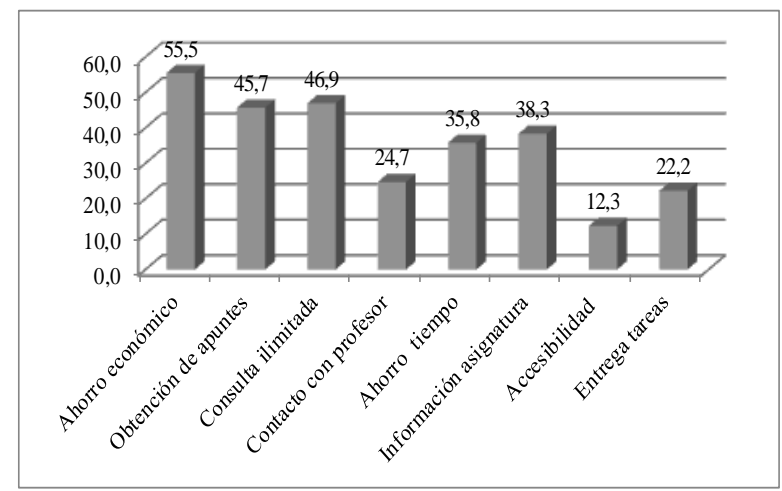

Figura 3. Principales ventajas de Moodle

Con respecto a las limitaciones o aspectos susceptibles de mejora de Moodle y/o derivados de su uso, los estudiantes consultados señalaron los fallos en la conexión a Internet (51.9\%), la obligatoriedad de disponer de Internet (48.1\%), así como los fallos en la aplicación (45.7\%). Por otra parte, un porcentaje inferior de estudiantes se refieren a al escaso uso de Moodle por parte de los docentes (13.6\%), la imposibilidad de hacer videochat entre profesor-alumno y/o entre compañeros (11.1\%), y al impedimento de cargar grandes archivos (2.5\%). Por último, cabe destacar que un $14.8 \%$ de los estudiantes no encuentra ningún tipo de limitación a Moodle.

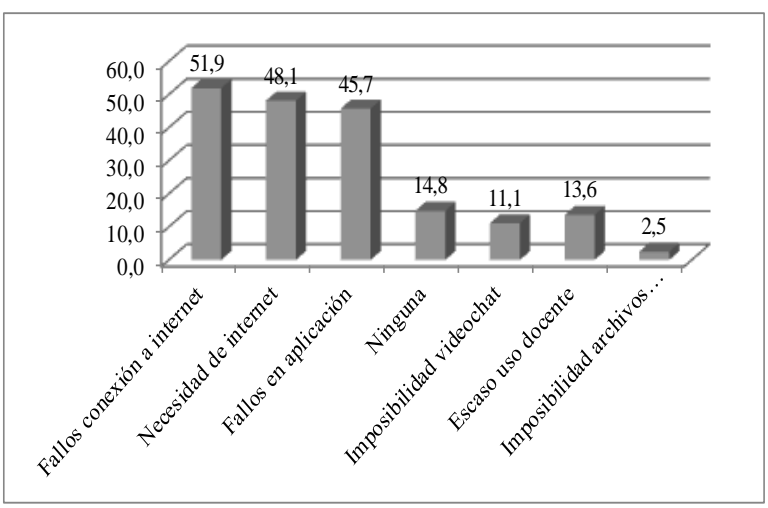

Figura 4. Principales limitaciones de Moodle

\section{Conclusiones}

A través de este artículo se ha pretendido conocer y analizar los usos que los estudiantes universitarios de segundo curso del grado de Educación Infantil de la Universidade da Coruña hacen sobre Moodle, así co mo sus percepciones sobre esta plataforma. Los resultados de este estudio pueden servir de cara a la mejora y desarrollo competencial de los estudiantes, así como a del profesorado.

De los resultados de este trabajo, en general podemos afirmar que los docentes de las asignaturas que curs an los 
alumnos consultados, incorporan la tecnología principalmente como un soporte de sus clases presenciales, empleando la plataforma Moodle como un repositorio de materiales y medio para recibir trabajos de los alumnos, y en menor medida co mo herramienta de comunicación a través del correo electrónico. Pero además, de este trabajo podemos extraer una serie de conclusiones más concretas que nos permiten situarnos con mayor conocimiento ante el papel desempeñan las nuevas tecnologías en la educación y la actitud de los alumnos respecto a las mismas:

- Pocos participantes han recibido formación específica sobre la plataforma Moodle. De hecho, demandan mayor formación sobre aspectos como el correo electrónico (quickmail), la utilización de las barras de herramientas y el funcionamiento general de la plataforma.

- El uso que hacen de Moodle es todavía muy limitado, puesto que lo suelen emplear exclusivamente para descargar apuntes. Estos datos coinciden con los datos arrojados por otros estudios (Sangrá, 2008; Marín y Amentia, 2009).

- La valoración global de Moodle es muy positiva, sobre todo por el ahorro económico que su utilización supone (no gastan dinero en fotocopias), aunque muchos participantes también ven limitaciones, haciendo referencia a aspectos técnicos, como los fallos de la conexión a internet o el hecho de que la plataforma se cuelgue o bloquee con demasiada frecuencia.

Es importante destacar, que aunque son múltiples los beneficios que aporta la plataforma Moodle tal y como señalaron los alumnos consultados en nuestro estudio, se debe hacer un buen uso de esta herramienta, recomendando que el alumno utilice la misma de forma crítica y que el docente la emp lee sin confundir el fin con los medios. Consideramos por lo tanto, que las TIC no son la solución principal de los problemas educativos, pero debemos aprovechar sus potencialidades de forma profunda y coherente, para obtener a través de las mis mas los mayores beneficios que brindan en la educación.

Tal y como lo plantean Díaz-Antón y Pérez (2005), hoy en día las Universidades presentan por lo generaluna necesidad urgente de alfabetización tecnológica, y de formación sobre las metodologías educativas aplicadas a la tecnología y nuevos modelos de evaluación. Según lo expresado por los alu mnos consultados, la Universidade da Coruña no se aleja de esta realidad, por lo que debe continuar formando a su alumnado para el uso de las TIC y de la plataforma "Moodle", ya que esta formación continua conllevará a generar cambios en la forma de pensar y actuar de los mismos, alcanzando mayores destrezas y competencias ante el uso de estas herramientas.

Para finalizar queremos resaltar, que son muchas las responsabilidades que tendrán que asumir los equipos directivos de las Universidades, para el logro del desarrollo de una docencia a través del uso intensivo de las TIC y de las plataformas de teleformación según los estándares de calidad, enfrentándose los mismos a una tarea compleja. Así, los docentes deberán ejercer la función de facilitador en el proceso de enseñanza/aprendizaje del alu mnado (Rodríguez, Varela, y Iseni, 2013). De esta forma, las Universidades responderán a un plan coherente, con una visión clara que coincida con el modelo de uso de las TIC que persiguen, fomentando la incorporación de estas herramientas para dar respuesta a las demandas de la sociedad de la información (Sangrá, 2008).

\section{Referencias}

Adell, J., Castellet, J. M. y Gumbau, J. P. (2004). Selección de un entorno virtual de enseñanza/aprendizaje de código fuente abierto para la Universitat Jaume I. Castellón: Centre d'Educacio i Noves Tecnologies de la Universitat Jaume I.

Ahn, J. Y. y Han, K. S. (2005). Web-based education: characteristics, problems, and some solutions. International Journal of Innovation and Learning, 2(3), 274-282.

Aydin, C. C. y Tirkes, G. (2010). Open Source Learning Management Systems in Distance Learning. Turkish Online Journal of Educational Technology, 9 (2), 175-184. http://www.tojet.net/articles/v9i2/9218.pdf

Bautista, G., Borges, F. y Forés, A. (2006). Didáctica universitaria en entornos virtuales de enseñanza-aprendizaje. Madrid: Narcea.

Díaz-Antón, G. y Pérez, M. (2005). Hacia una ontología sobre LMS. Actas de las VII Jornadas Internacionales de las Ciencias Computacionales (pp. 1-7). Colima: Universidad de Colima. http://www.lisi.usb.ve/publicaciones/02\%20calidad\% 20sistemica/calidad_59.pdf

Escobar, T y Monge, P. (2012). The acceptance of Moodle technology by business administration students. Computers \& Education, 58 (4), 1085-1093. http://www.sciencedirect.co m/science/article/pii/S036 0131511002934

Henríquez, G.; Ugel, E. E. (2012). Migración de lo presencial a lo virtual en la asignatura introducción a la computación del programa de enfermería de la UCLA. RIED. Revista Iberoamericana de Educación a Distancia, 15(1), 127-142. http://dx.doi.org/10.5944/ried.1.15.780

Marín, F. y A mentia, J.I. (2009). Los estudiantes frente al reto de las TIC en la universidad. Moodle y eKasi en la Facultad de Ciencias Sociales y de la Comunicación (Universidad del País Vasco). Revista de estudios de comunicación - ZER, 27(14), 319-347. http://www.ehu.es/zer/heme roteca/pdfs/zer27-15-mari n.pdf

Martín, B. y Rodríguez, D. (2012). La evaluación de la formación universitaria se mipresencial y en línea en el contexto del EEES mediante el uso de los informes de actividad de la plataforma Moodle. RIED. Revista Iberoamericana de Educación a Distancia, 15(1), 159-178. http://dx.doi.org/10.5944/ried.1.15.782 
Martín, T. y Serrano, A. (2009). The role of new technologies in the learning process: Moodle as a teaching tool in Physics. Computers \& Education, 52, 35-44.

http://www.sciencedirect.co m/science/article/pii/S036 013150800095X

Martínez, C. y Fernández, M. S. (2011). El uso de Moodle como entorno virtual de apoyo a la enseñanza presencial. En R. Roig y C. Laneve (Coord.). La práctica educativa en la Sociedad de la Información: Innovación a través de la investigación (pp. 291-300). Alcoy: Marfil.

Ministerio de Educación, Cultura y Deporte (mec) (2006). Propuestas para la renovación de las metodologías educativas en la universidad. http://sestud.uv.es/varios/ope/PROPUESTA_RENOV ACION.pdf

Oliveros, L. (2006). Identificación de competencias: una estrategia para la formación en el Espacio Europeo de Educación Superior. Revista Complutense de Educación, 17(1), 101-118.

$\mathrm{http}: / /$ revis tas.ucm.es/index.php/RCED/article/view/R CED0606120101A

Peat, M., y Franklin, S. (2002). Supporting Student Learning: The Use of Computerbased 9. Formative Assesment Modules. British Journal of Educational Technology, 33(5), 515-523.

Perkins, M. y Pfaffman, J. (2006). Using a course management system to improve classroom communication. Science Teacher, 73(7), 33-37.

Richardson, J. y Swan, K. (2003). Examining social presence in online courses in relation to students' perceived learning and satisfaction. Journal of Asynchronous Learning 6 (1), 21-40.
Rodríguez, M., Varela, J. e Iseni, A. (2013). Virtual learning in higher education. Anglisticum Joumal, 2(4), 262-278.

http://www.ifets.info/journals/7_4/21.pdf

Saito, D. S. y Ulbricht, V. R. (2012). Learning Managent Systems and Face-to-Face Teaching in Bilingual Modality (Libras/Portuguese). IEEE Latin America Transactions, 10(5), 2168-2174.

Sánchez, J., Sánchez, P. y Ramos, F.J. (2012). Usos pedagógicos de Moodle en la docencia universitaria desde la perspectiva de los estudiantes. Revista Iberoamericana de Educación, 60, 15-38. http://www.rieoei.org/rie60a01.pdf

Sangrá, A. (2008). La Integració de les Tic a la Universitat: Models, Problemes I Reptes. Tesis doctoral, Departamento de Pedagogía, Universitat Rovira I Virgili, España, Tarragona, España. http://tecnologiaedu.us.es/nweb/htm/pdf/sangra.pdf

Swan, K., Shea, P., Fredericksen, E., Pickett, A., Pelz, W. y Maher, G. (2000). Building Knowledge Building Communities: Consistency, Contact and Communication in the Virtual Classroom. Journal of Educational Computing Research, 23(4), 359-83.

Williams van Rooij, S. (2012). Open-source learning management systems: a predictive model for higher education. Journal of Computer Assisted Learning, 28 (2), 114-125.

http://onlinelibrary.wiley.co m/doi/10.1111/j.1365-272 9.2011.00422.x/abstract 